\title{
A Modified Non Linear Median Filter for the Removal of Medium Density Random Valued Impulse Noise
}

\author{
Govind Ballabh Khan \\ M.Tech Student \\ OIST Bhopal, M.P.
}

\author{
Sandeep Pratap Singh \\ Dept. of Computer Science \\ OIST Bhopal, M.P
}

\begin{abstract}
In the field of image processing, elimination of noise from digital images plays a vital role. Effective detection of noisy pixel based on median value and an efficient algorithm for the estimation and replacement of noisy pixel has been carried out in this proposed method in which replacement of noisy pixel is carried out twice, which results in better preservation of image details. The presence of high performing detection stage for the detection noisy pixel makes the proposed method suitable in the case of high density random valued impulse noise, hence the proposed method yields better image quality with improved peak signal to noise ratio (PSNR) and reduced mean square error (MSE). Results of proposed method has been compared with many other standard median based switching filters in terms of visual and quantitative measures and the performance of the proposed method is presented.
\end{abstract}

\section{Keywords}

Mean square error, Peak signal to noise ratio, Random valued impulse noise; switching median filter.

\section{INTRODUCTION}

Preservation of digital images during the process of image acquisition or transmission has always been a very cumbersome task for researchers. Images are often corrupted by the impulse noise, Gaussian noise, shot noise, speckle noise etc. Preservation of image details and suppression of noise are the two important aspects of image processing. Generally impulse noise is classified into two types: the saltand-pepper noise also known as the fixed valued impulse noise and the random-valued impulse noise. Here in this paper we focus on random valued impulse noise. Random valued impulse noise will produce impulses whose gray level value lies within a predetermined range. The random value impulse noise lies between 0 and 255 and it is very difficult to remove this noise. Salt and pepper noise is sometime called as Fixed valued impulse noise producing two gray level values 0 and 255 . Where 0 value belongs to black and 255 belongs to white on the gray scale. It is generally reflected by pixels having minimum and maximum value in gray scale image. Generally the basic idea behind image de-noising is the detection stage which identifies the noisy and noise free pixels of the corrupted image, after that noise removal part removes the noise from the corrupted image under process while preserving the other important detail of image.

There are two types of filters in spatial domain: linear filter and non-linear filter. Linear filters are like wiener filter, mean filter. Here we propose a nonlinear median filter which removes random valued noise and preserves the edges of image. Initially standard median filter was used but later on switching based median filters were developed which provides better results. Some other result oriented standard median filters were Developed, like weighted median filter, SDROM filter [8], centre weighted median filter [14], adaptive median filter, rank conditioned rank selection filter [12] and many other improved filters. The consequences of median filter also depend on the size of filtering window. Larger window has great noise suppression capability but image details (edges, corners, fine lines) preservation is limited, while a smaller window preserves the details but it will cause the reduction in noise suppression. Noise detection is a vital part of a filter, so it is necessary to detect whether the pixel is noisy or noise free. Only noisy pixels are subjected to de-noising and noise free pixels remains untouched.

\section{NOISE MODEL}

Random valued impulse noise will produce impulses whose gray level value lies within a predetermined range. The random value impulse noise lies between 0 and 255 and it is very difficult to remove this noise.

Impulse noise is modeled as:

$$
f(y)=\left\{\begin{array}{c}
\frac{q}{2} \text { for } \ldots y=0 \\
1-q \text { for } \ldots y=T(i, j) \\
\frac{q}{2} \text { for } \ldots y=255
\end{array}\right.
$$

Here $f(y)=$ probability density function,

$$
\begin{aligned}
& T(i, j)=\text { intensity value, } \\
& Y(i, j)=\text { Noisy pixel, } \\
& q=\text { Noise density. }
\end{aligned}
$$

\section{RELATED WORK}

In the spatial domain the most basic nonlinear filter is the standard median filter (SMF)[5]. It replaces each pixel in the image by the median value of the corresponding filtering window. The standard median filter works effectively for low noise densities but at the cost of blurring the image.

Consider that the pixel values in neighborhood are taken in to sequence M1, M2, M3 ............Mn.

To calculate the median value of pixels, first all pixels are sorted either in ascending or descending order. After sorting these pixels, the sequence will be $\mathrm{Mi} 1 \leq \mathrm{Mi} 2 \leq \mathrm{Mi} 3 \leq \ldots \ldots \ldots \ldots \ldots . .$. Min, in ascending order and Mi1 $\geq$ $\mathrm{Mi} 2 \geq \mathrm{Mi} 3 \geq \ldots \ldots \ldots \ldots . . . . . M i n$, in descending order.

Thus, mathematically median is expressed as: []

$$
\begin{aligned}
\operatorname{Median}(\mathrm{M}) & =\operatorname{Med}\{\mathrm{Mi}\} \\
& =\left\{\begin{array}{l}
\operatorname{Mi}(\mathrm{n}+1) / 2, \quad \mathrm{n} \text { is odd } \\
1 / 2[\operatorname{Mi}(\mathrm{n} / 2)+\operatorname{Mi}(\mathrm{n} / 2)+1], \mathrm{n} \text { is even }
\end{array}\right.
\end{aligned}
$$

n' is generally odd. 


\section{Signal-dependent rank ordered mean filter (SD-ROM) [8]:}

It is an efficient nonlinear algorithm to suppress impulse noise from highly corrupted images while preserving image details and features. The method is applicable to all impulse noise models, including fixed valued (equal height or salt and pepper) impulses and random valued (unequal height) impulses, covering the whole dynamic range. The technique achieves excellent tradeoff between the suppression of noise, and preserving the details and edges without undue increase in computational complexity.

\section{Rank Conditioned Rank Selection Filter (RCRS) [12]:}

The RCRS filters are proposed in the general structure of rank selection filters. The information utilized by RCRS filters is the ranks of selected input samples; hence the name rank conditioned rank selection filters. The number of input sample rank used in this decision is referred to as the order of RCRS filter. The order ranges from zero to the number of samples in the observation window, giving the filters valuable flexibility. Low-order filters can give good performance and are relatively simple to optimize and implement.

\section{Progressive Switching Median Filter (PSM) [9]:}

It is a median-based progressive switching median (PSM) filter, proposed for the Removal of Impulse Noise from Highly Corrupted Images. The filtering method is based on the following two main schemes: (1) Switching scheme : An impulse detection scheme is used before filtering, thus only a fraction of all the pixels will be subjected to filtering process and (2) Progressive methods : Both the impulse detection and the noise filtering procedures are progressively applied through a number of iterations. The main advantage of this method is that some impulse pixels located in the middle of large noise blotches can also be properly detected and filtered, which results in better restoration, especially for the cases where the images are highly corrupted.

In 2007 Yiqiu Dong and Shufang Xu [6], proposed a new impulse detector, which utilize the differences between the current pixel and its neighbors aligned with four foremost directions. After impulse detection, the filter simply do not replace noisy pixels identified by outputs of median filter, but continue to make use of the information of the four directions to weight the pixels in the window so as to preserve the details of image.

However; further lessening in computational complexity is desired. Here we proposed a method with computational simplicity which makes it enable to restore images at faster rate.

In 2014, Aparna Sarkar, Suvmoy Changder and J.K. Mandal [1], proposed a random value impulse noise removal in thermal images. Proposed a new method threshold based Directional Weighted Median (TDWM) Filter for Removal of Random valued Impulse Noises (RVIN) from digital and Infrared Images, has been proposed. This filter works in two phases; at first it detects the impulses, and then replaces the pixels affected with impulses by an estimated value. This filter works efficiently in suppressing impulses from both digital images and infrared images highly corrupted with random valued impulses. Extensive simulations show that proposed filter provides better results than existing filters in suppressing impulses.
The main drawback of this paper used a $5 * 5$ window size for the removal of impulse noise. In the $5 * 5$ window size contain 25 pixels and the size of window is big so generate the bluer. Blur the main problem in the previous work. In the high noise density removal contain the blur of images in restoration.

\section{PROPOSED WORK}

The feature of proposed switching median filter is described in this section which shows the efficient de-noising of highly corrupted images. The algorithm consists of two stages. In the first stage, the noisy pixels are detected by two dynamically calculated threshold determined by median of each row, column and both diagonals. It's ability to detect noisy pixel with very good accuracy even in the presence of multiple impulses within the sliding window.

In the second stage, if a pixel is considered to be noisy, it is substituted by performing a nonlinear prediction from the neighbourhood pixels in the current window by calculating median prior to estimation. Then the noisy pixel is replaced by the median value of the pixels within the current window. The sliding window can be assumed $3 \mathrm{X} 3$ matrix, which has three rows, three columns and two diagonals as shown below:

Table I Filtering window of size $3 \times 3$

\begin{tabular}{|c|c|c|c|}
\hline & Column 1 & Column 2 & Column 3 \\
\hline Row 1 & $\mathrm{X}_{1}$ & $\mathrm{X}_{2}$ & $\mathrm{X}_{3}$ \\
\hline Row 2 & $\mathrm{X}_{4}$ & $\mathrm{X}_{5}$ & $\mathrm{X}_{6}$ \\
\hline Row 3 & $\mathrm{X}_{7}$ & $\mathrm{X}_{8}$ & $\mathrm{X}_{9}$ \\
\hline
\end{tabular}

\section{The proposed method executes in the following steps :}

Step 1: Read the corrupted image and select a sliding window size of $3 \times 3$.

Step 2: For each window, calculate median value of each row, column and both diagonals which are denoted as Row $\left(\mathrm{M}_{\mathrm{R} 1}\right.$, $\left.\mathrm{M}_{\mathrm{R} 2}, \mathrm{M}_{\mathrm{R} 3}\right)$, Column $\left(\mathrm{M}_{\mathrm{C} 1}, \mathrm{M}_{\mathrm{C} 2}, \mathrm{M}_{\mathrm{C} 3}\right)$, and both Diagonals $\left(\mathrm{M}_{\mathrm{D} 1}, \mathrm{M}_{\mathrm{D} 2}\right)$.

Step 3: Calculate maximum threshold $\left(\mathrm{Th}_{\max }\right)$ and minimum threshold $\left(\mathrm{Th}_{\min }\right)$ using the medians calculated in the step 2.

$\mathrm{Th}_{\max }=\max \left[\mathrm{M}_{\mathrm{R} 1}, \mathrm{M}_{\mathrm{R} 2}, \mathrm{M}_{\mathrm{R} 3}, \mathrm{M}_{\mathrm{C} 1}, \mathrm{M}_{\mathrm{C} 2}, \mathrm{M}_{\mathrm{C} 3}, \mathrm{M}_{\mathrm{D} 1}, \mathrm{M}_{\mathrm{D} 2}\right]$
$\mathrm{Th}_{\min }=\min \left[\mathrm{M}_{\mathrm{R} 1}, \mathrm{M}_{\mathrm{R} 2}, \mathrm{M}_{\mathrm{R} 3}, \mathrm{M}_{\mathrm{C} 1}, \mathrm{M}_{\mathrm{C} 2}, \mathrm{M}_{\mathrm{C} 3}, \mathrm{M}_{\mathrm{D} 1}, \mathrm{M}_{\mathrm{D} 2}\right]$

Step 4: Check whether the value of target pixel lies between

$\mathrm{Th}_{\max }$ and $\mathrm{Th}_{\min }$

$$
\mathrm{Th}_{\max }>\mathrm{X}_{5}>\mathrm{Th}_{\min }
$$

- If the above condition is true then it is considered as noise free pixel and goes to Step 2 .

- If the above condition is false then the pixel is treated as noisy pixel and proceeds to Step 5 .

Step 5: Calculate the median value of current window.

Step 6: Replace the targeted noisy pixel $\left(\mathrm{X}_{5}\right)$ by the median value calculated in step 5 .

Step 7: If the whole image is not processed, go to step 2 otherwise proceed to step 8 .

Step 8: Stop. 


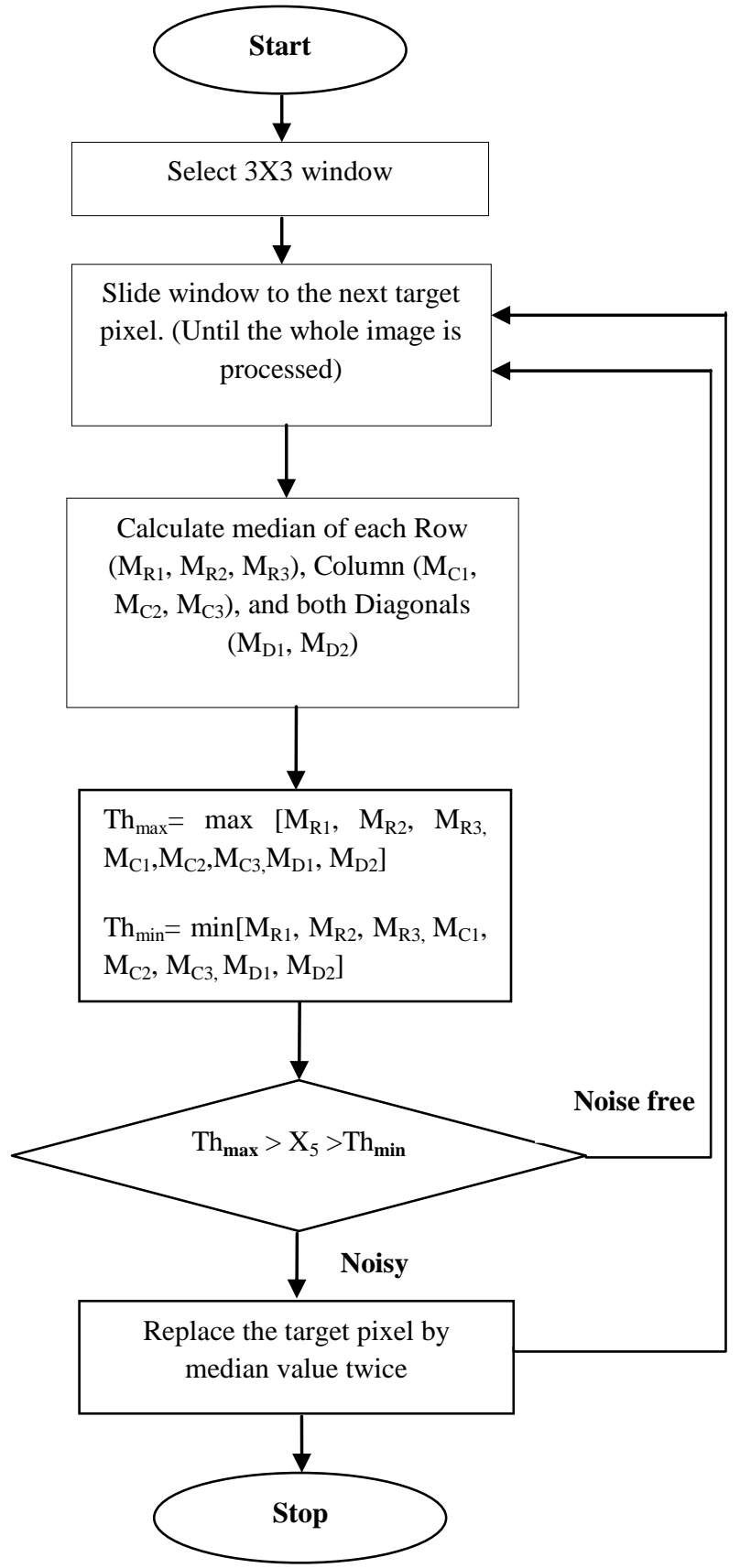

Fig.1. Flow chart of proposed method

\section{SIMULATIONS AND RESULTS}

We performed this method on Matlab R-2012 b results are shown in table II, table III and figure 2. This method yields better results for high density noisy images. The simulation result shows that PSNR (Peak Signal to Noise Ratio) of denoised image by this filter is better than other filters.

The PSNR is expressed as:

$$
P S N R=\operatorname{lolog}_{10} \frac{(255)^{2}}{\mathrm{MSE}}
$$

Where MSE (Mean Square Error) is

$$
M S E=\frac{\sum_{i=1}^{m} \sum_{j=1}^{n}\{Z(i, j)-A(i, j)\}^{2}}{m \times n}
$$

Table of IR image Enhancement

\begin{tabular}{|c|c|c|c|}
\hline $\begin{array}{c}\text { Noise } \\
\text { Density }\end{array}$ & Image Type & $\begin{array}{c}\text { Image3 } \\
\text { PSNR(dB) }\end{array}$ & $\begin{array}{c}\text { Proposed } \\
\text { Method }\end{array}$ \\
\hline $10 \%$ & Restored image & 26.85 & 30.9 \\
\hline $20 \%$ & Restored image & 24.92 & 29.19 \\
\hline $30 \%$ & Restored image & 23.66 & 27.18 \\
\hline $40 \%$ & Restored image & 22.54 & 25.03 \\
\hline $50 \%$ & Restored image & 21.56 & 22.77 \\
\hline $60 \%$ & Restored image & 20.69 & 20.78 \\
\hline
\end{tabular}

The above table shows the thermal image comparision on different noise density.

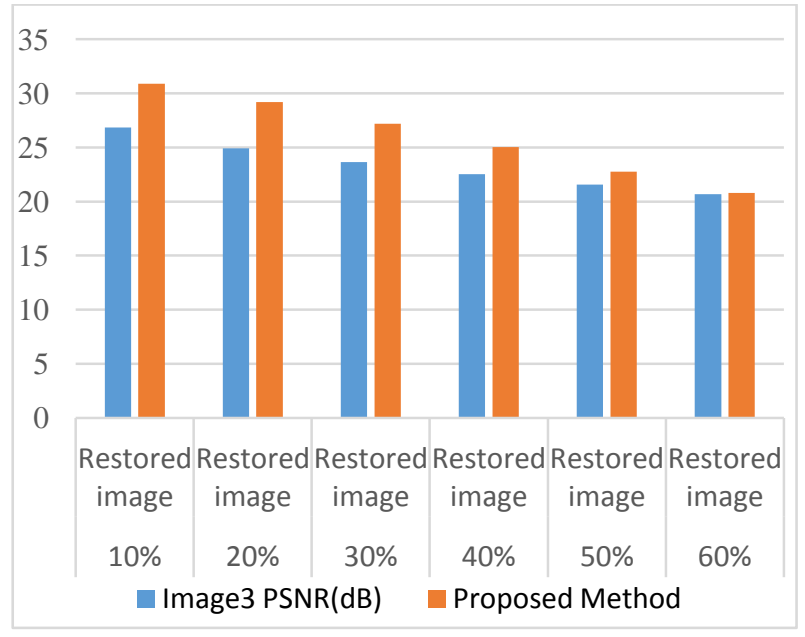

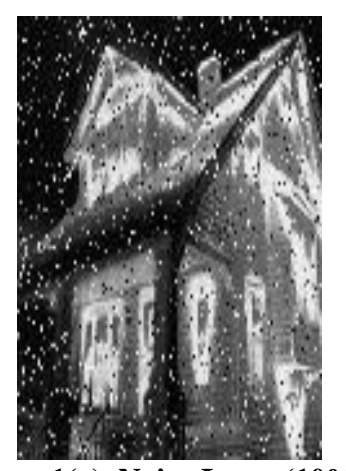

1(a). Noisy Image(10\%)

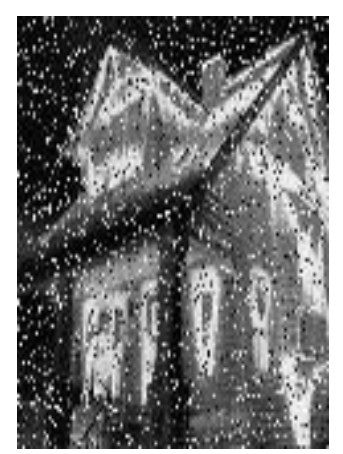

1(c). Noisy Image(20\%)

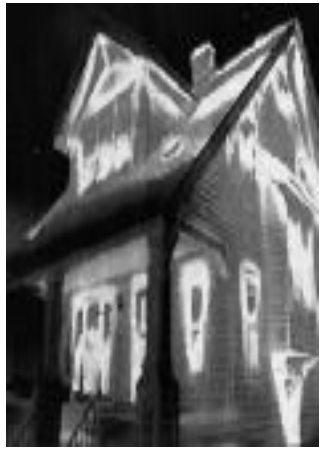

1(b). Restored Image

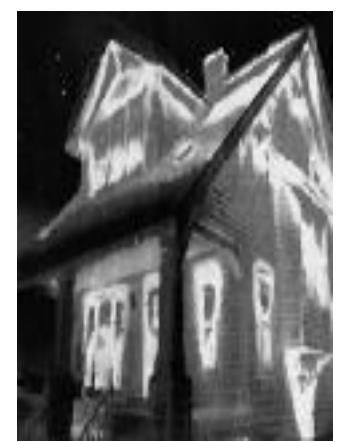

1(d). Restored Image 


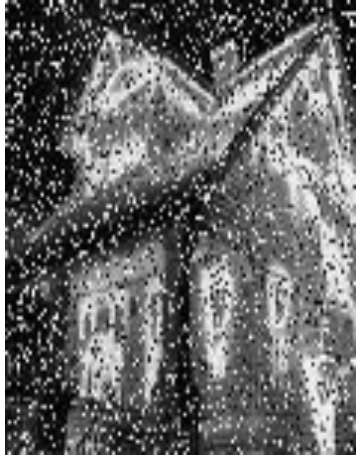

1(e).Noisy Image $(30 \%)$

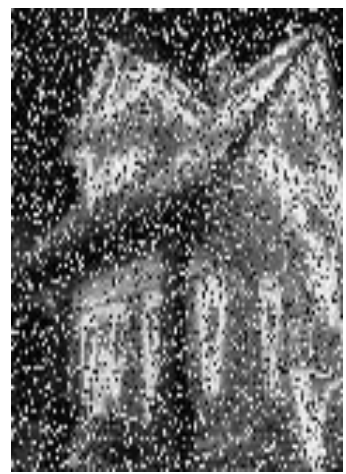

1(g). Noisy Image(40\%)

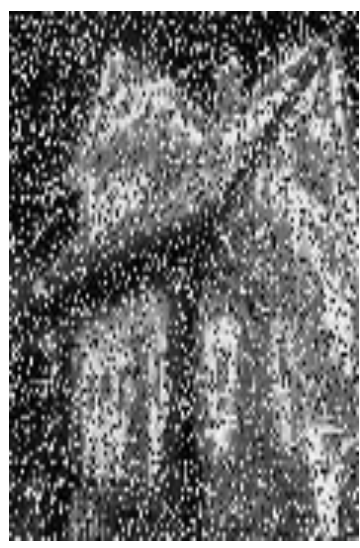

1(i). Noisy Image $(50 \%)$

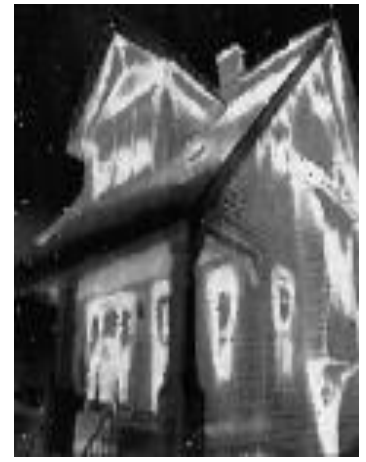

1(f). Restored Image

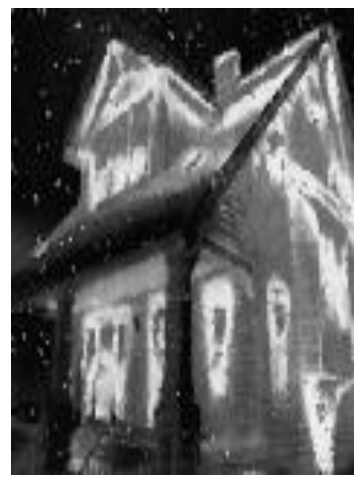

1(h). Restored Image

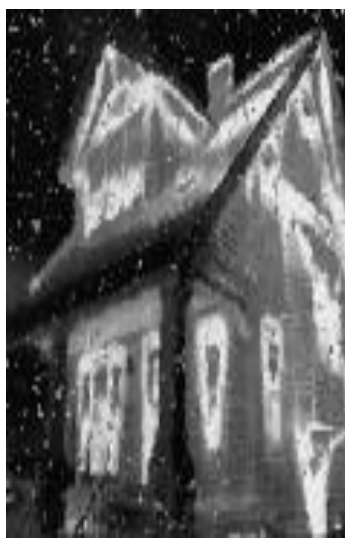

1(j). Restored Image
The results in the Table II clearly show that the PSNR of proposed method is better at high density random valued impulse noise. The results are compared with several standard median based filters like Median filter (MF)[5], Centre weighted median filter (CWM)[14], Progressive switching median filter (PSM)[9], Signal dependent rank order median filter (SDROM)[8], Recursive adaptive centre weighted median filter (RACWM), Tri-state median filter (TSM)[12]. This method is tested on Lena image of size 256X256 shown in fig.2. The fig 2(b), 2(d), 2(f), 2(h), 2(j) shows Lena image corrupted by $50 \%, 60 \%, 70 \%, 80 \%$ and $90 \%$ respectively and figure 2(b), 2(d). 2(f), 2(h), 2(j) show images De-noised by proposed method.
Table II Comparison of PSNR values of different filters for LENA Image

\begin{tabular}{|c|c|c|c|c|r|}
\hline Filters & \multicolumn{5}{|c|}{ PSNR For Restored image in dB “Leena } \\
Image " \\
\hline & $10 \%$ & $20 \%$ & $30 \%$ & $40 \%$ & $50 \%$ \\
\hline ROAD & 34.79 & 28.58 & 23.72 & 20.12 & 17.38 \\
\hline ROLD & 30.05 & 26.87 & 23.08 & 19.87 & 17.29 \\
\hline SD & 30.93 & 27.3 & 23.81 & 20.8 & 18.12 \\
\hline ROR & 31.83 & 28.36 & 24.93 & 21.51 & 18.69 \\
\hline RDD & 32.11 & 29.9 & 26.14 & 22.98 & 19.99 \\
\hline PWMAD & 34.36 & 30.58 & 25.95 & 22.41 & 19.42 \\
\hline ACWM & - & 36.07 & 32.59 & 28.79 & 25.19 \\
\hline AMP & 28.06 & 26.79 & 24.03 & 23.17 & 21.99 \\
\hline $\begin{array}{l}\text { Base } \\
\text { paper }\end{array}$ & 31.59 & 29.51 & 28.02 & 26.85 & 25.88 \\
\hline Proposed & 31.0 & 30.01 & 28.89 & 27.01 & 26.05 \\
\hline
\end{tabular}

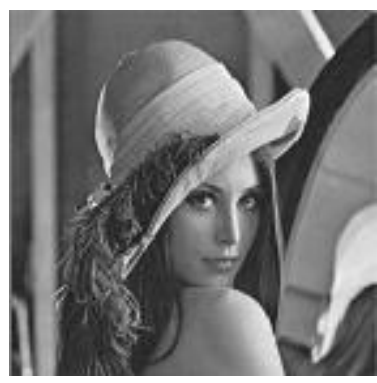

2(a). Original Lena image

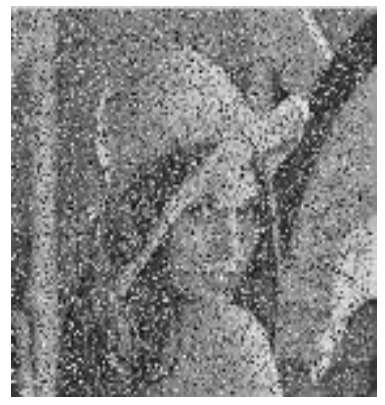

2(b). $50 \%$ noisy image

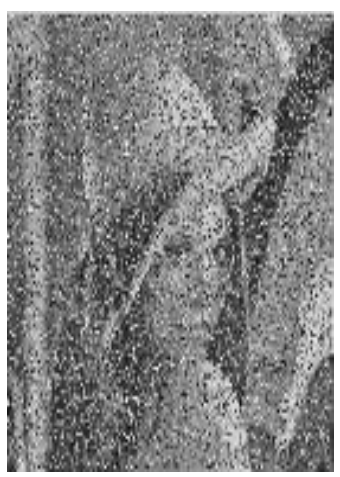

2(d). $60 \%$ noisy image

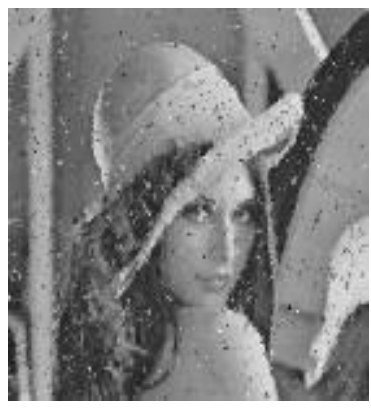

2(c). Restored image

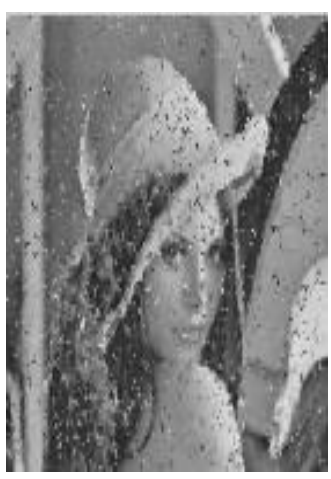

2(e). Restored image 


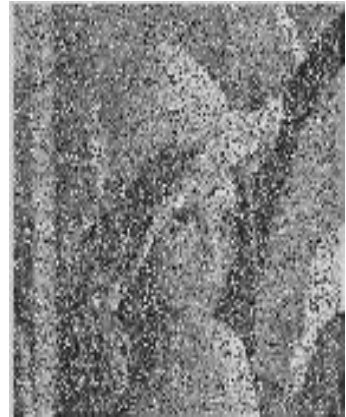

2(f). $70 \%$ noisy image

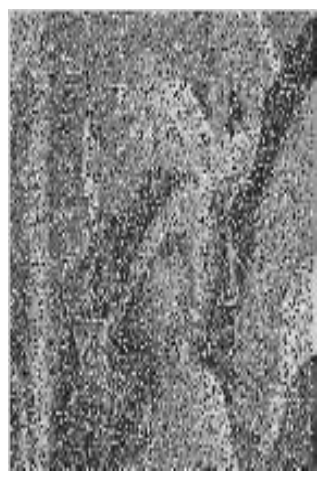

2(h). $80 \%$ noisy image

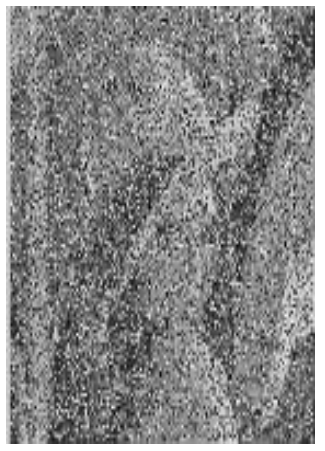

2(j). $90 \%$ noisy image

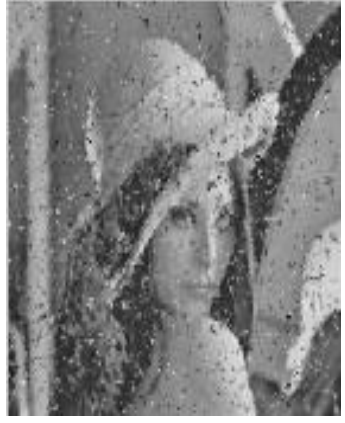

2(g). Restored image

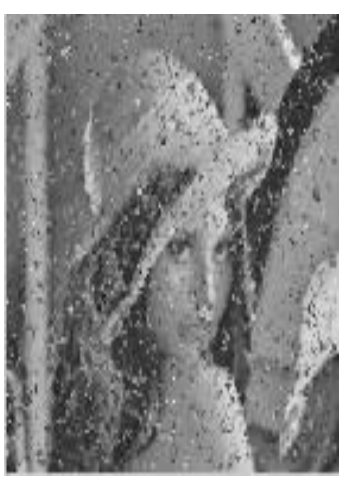

2(i) Restored image

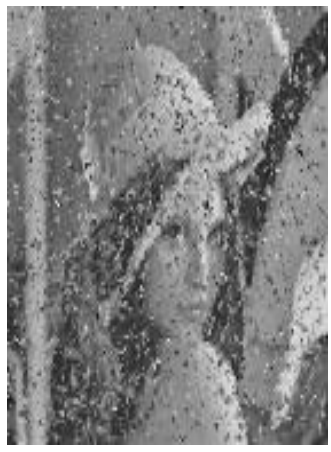

2(k). Restored image
Fig 2. Experimental Results of Proposed Method for Lena Image

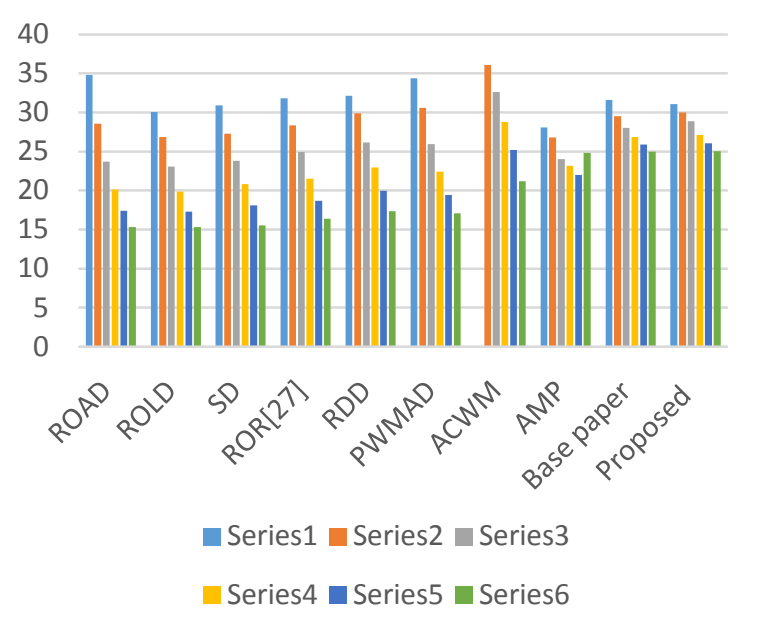

Fig. 3. Graphical representation of PSNR of different standard filters at different noise density
Table III .Different Parameter Calculated for "Govind Image"

\begin{tabular}{|l|l|l|l|l|}
\hline & \multicolumn{4}{|c|}{ Different parameter Calculated For "Govind } \\
& \multicolumn{4}{|c|}{ Image” } \\
\hline $\begin{array}{l}\text { Noise } \\
\text { Density }\end{array}$ & TIME & MSE & PSNR & RMSE \\
\hline $10 \%$ & 8.847001 & 11.8800 & 37.3827 & 3.4467 \\
\hline $20 \%$ & 9.551353 & 18.9325 & 35.3587 & 4.3512 \\
\hline $30 \%$ & 9.575893 & 39.7902 & 32.1330 & 6.3079 \\
\hline $40 \%$ & 9.192469 & 104.2036 & 27.9520 & 10.2080 \\
\hline $50 \%$ & 9.718122 & 210.6728 & 24.8947 & 14.5146 \\
\hline $60 \%$ & 8.493376 & 355.3040 & 22.6248 & 18.8495 \\
\hline $70 \%$ & 11.085565 & 599.5087 & 20.3528 & 24.48 \\
\hline $80 \%$ & 19.304884 & 893.3131 & 18.6208 & 29.8883 \\
\hline $90 \%$ & 8.889668 & $1.2045 E+03$ & 17.3228 & 34.7056 \\
\hline
\end{tabular}
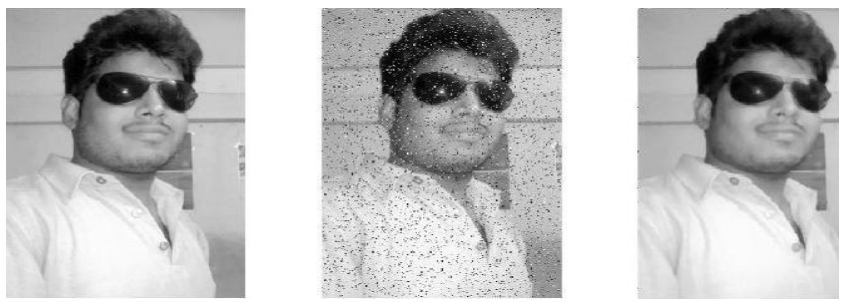

4(a)Original Image 4(b) 10\% Noisy Image 4(c) Restored Image
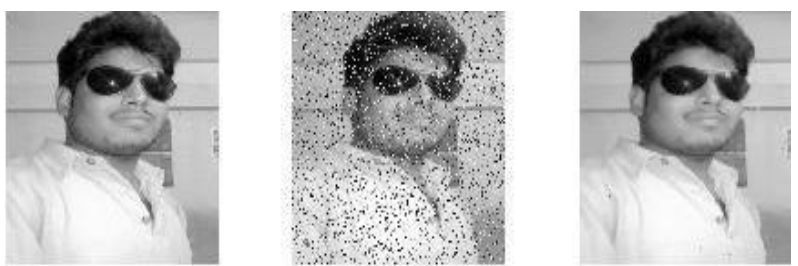

4(d)Original Image 4(e) 20\% Noisy Image 4(f) Restored Image
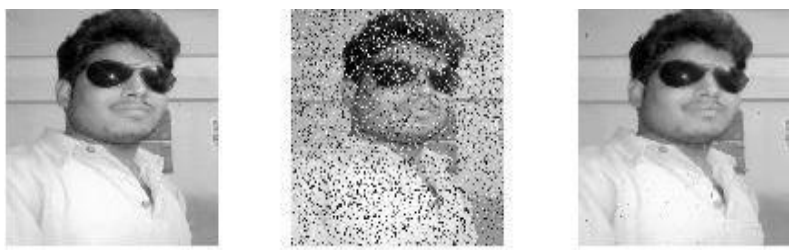

4(g)Original Image 4(h) 30\% Noisy Image 4(i) Restored Image
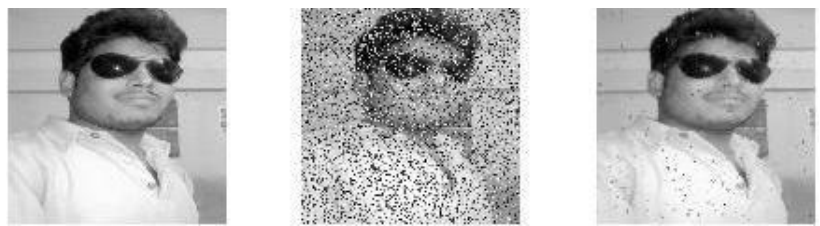

4(j)Original Image 4(k) 40\% Noisy Image 4(l) Restored Image 

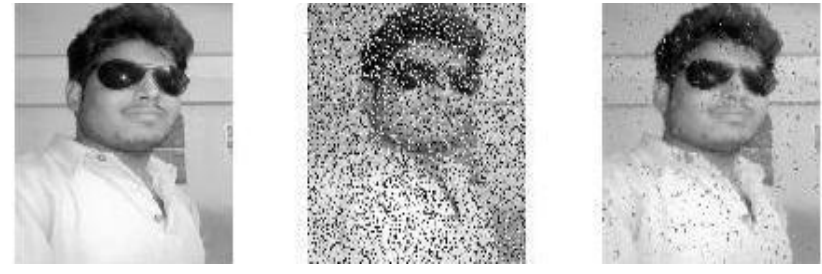

4(m)Original Image 4(n) 50\% Noisy Image 4(o) Restored Image
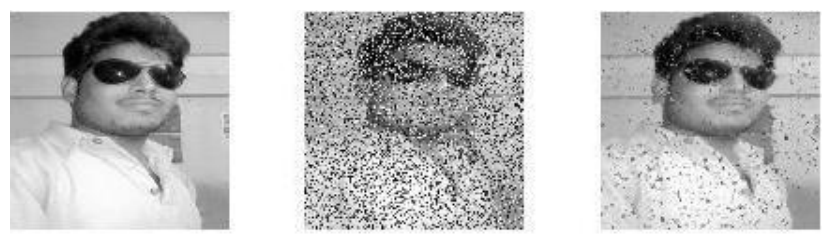

4(p)Original Image 4(q) $60 \%$ Noisy Image 4(r) Restored Image

\section{CONCLUSION}

The proposed filtering method yields better results for high density random valued impulse noise. Effective detection of noisy pixel results in better restoration of image and preservation of the image details. In this paper dual median filtering is used for improving peak signal to noise ratio (PSNR) and reducing mean square error (MSE) values. Computational simplicity of this filter makes it enable to restore images at faster rate. The performance of this method depends on two dynamically changing threshold values which adaptively change according to the noise density. The experimental results show the proposed methodology performs better than other several existing methods.

\section{REFERENCES}

[1] E. Abreu and S. Mitra, "A signal-dependent rank ordered mean (SDROM) filter-a new approach for removal of impulses from highly corrupted images," in ICASSP, vol. 4, pp. $2371-2374,1995$ IEEE.

[2] Z. Wang and D. Zhang, "Progressive switching median filter for the removal of impulse noise from highly corrupted images," IEEE Trans. on Circuits and Systems II: Analog and Digital Signal Processing, vol. 46, no. 1, pp. 78-80, 1999.

[3] R. C. Hardie and K. E. Barner, " Rank-conditioned rank selection filters for signal restoration," IEEE Transactions on Image Processing, vol. 2 pp. 192-206, 1994

[4] Y. Dong and S. Xu, "A new directional weighted median filter for removal of random-valued impulse noise,"
IEEE Signal Processing Letters, vol. 14, no.3, pp. 193196, 2007.

[5] Guoping Qiu, “ An improved Recursive Median Filtering Scheme for Image Processing", IEEE Trans. on Image Processing, Vol.5, No.4, pp. 646-648. 11996.

[6] T. Chen, K.-K. Ma, and L.-H. Chen, "Tri-state median filter for image denoising," IEEE Trans. Image Processing, vol. 8, no. 12, pp. 1834-1838, 1999.

[7] Xiaoyin Xu, Eric L. Miller, Dongbin Chen and Mansoor Sarhadi "Adaptive two-pass rank order filter to remove impulse noise in highly corrupted images", IEEE Transactions on Image Processing, Vol. 13, No. 2, February 2004.

[8] T. Song, M. Gabbouj, and Y. Neuvo, "Center weighted median filters: some properties and applications in image processing," Signal Processing, vol. 35, no. 3, pp. 213 229, 1994.

[9] S.-J. Ko and Y. Lee, "Center weighted median filters and their applications to image enhancement," IEEE Transactions on Circuits and Systems, vol. 38, no. 9, pp. 984-993, September 1991.

[10] J. Bhabesh Deka and Dipranjan Baishnab "A Linear Prediction Based Switching Median Filter for the Removal of Salt and Pepper Noise from Highly Corrupted Image" in CISP2012 @2012 IEEE.

[11] Shanmugavadivu P \& Eliahim Jeevaraj P S, "Laplace Equation based Adaptive Median Filter for Highly"CorruptedImages",International Conference on Computer Communication and Informatics (ICCCI 2012), 2012.

[12] Dagao Duan, Yueliang Wan, "A Detail Preserving Filter for Impulse Noise Removal" in International Conference on Computer Application and System Modeling (ICCASM 2010).

[13] Gonzalez R.C, Woods. R.E., Digital Image Processing, $3^{\text {rd }}$ edition, Pearson Prentice Hall, 2009.

[14] Aparna Sarkar, Suvamoy Changder,and J.K.Mandal"A Threshold based Directional Weighted MedianFilter for Removal of Random Impulses in Thermal Images" IEEE-2014(ICBIM)

[15] T. Chen and H.Wu Adaptive impulse detection using centre-weighted median filters, Signal Processing Letter., vol.8, no. 1, pp. 13, Jan. 2001 